\title{
Bird Species Diversity and Abundance in the Abiriw Sacred Grove in the Eastern Region of Ghana
}

\author{
L. Kangah-Kesse' ${ }^{1}$, D. Attuquayefio ${ }^{1 *}$, E. Owusu ${ }^{2}$, F. Gbogbo ${ }^{1}$ \\ ${ }^{1}$ Department of Zoology, University of Ghana, P. O. Box LG 67, Legon-Accra, Ghana \\ ${ }^{2}$ Ghana Wildlife Society, Accra, Ghana \\ *Corresponding author
}

\begin{abstract}
The sacred grove concept is one of the strategies developed by many human societies to conserve biological resources using a traditional approach. Sacred groves are currently under threat from anthropogenic activities due to lack of enforcement of traditional edicts to check encroachment. The birds inhabiting the Abiriw Sacred Grove in the Akwapim North District in the Eastern Region of Ghana were surveyed between February and April 2005 to determine the current ecological status of the grove, and to establish a bird species list. The transect count method was used to sample the birds in the grove. A total of 411 individual birds belonging to 22 families and 66 species was recorded, out of which 211 individuals of 41 species occurred in the forest-cultivated land boundary, 111 (36 species) in pristine forest, and 89 (40 species) in secondary forest. A significant proportion of species in the grove were savanna specialists. Similarity indices indicated different levels of degradation of the various blocks. The current situation needs immediate attention to stem the tide of fragmentation and degradation.
\end{abstract}

\section{Introduction}

Throughout the ages, traditional African societies have maintained complex religious and cultural belief systems that guided the conservation of biodiversity using traditional norms such as taboos, totems and myths. Such traditional practices enabled the protection of biological resources from human disturbances and wanton over-exploitation. The establishment of sacred groves, defined as small patches or islands of remaining original habitat (Kingdon, 1989), or forests of various dimensions partially- or fully-protected by local religious and/or cultural agents (Campbell, 2004) ensured the protection of forests surrounding environmentally-sensitive areas, and ranged in size from hundreds of hectares of forest to small areas of about 0.5 ha containing single trees or a few stones (Gordon, 1992).

Because of their perceived links to some deities or ancestral spirits, sacred groves are referred to variously as nananompow (ancestral grove or royal mausoleum) (Adarkwa-Dadzie, 1997), abosompow or asoneyeso (shrine), mpanyinpow (ancestral forest), and nsamanpow (burial grounds) by the Akans (BSP, 1993; Ntiamoa-Baidu, 1995). Sacred groves serve important ecological and socio-cultural functions by preserving virgin forests, being important refuges for rare and useful local biodiversity, and being sources of herbs for medicinal, social and religious purposes (Dorm-Adzobu et al., 1991; Decher, 1997). There are an estimated 2,000-3,200 sacred groves in Ghana, about $80 \%$ of which occur in the southern half of the country (Gordon, 1992).

Because of the linkage between traditional beliefs and deities or ancestral spirits, disobedience or disregard of traditional laws (taboos) attracts severe punishments for culprits, and high prices of atonement, including making sacrifices and performing certain rites to avert any mishaps, illhealth, and death. Unfortunately, this has not been effective deterrent for the reckless use of biological resources by local communities. Today, the traditional belief systems, which were fundamental to the concept of sacred grove conservation, are considered mere superstitions, with the rituals now known to very few people, mostly of the older generation. This situation could be attributed to (i) rapid population growth and its attendant problems of urbanization, migration, and resettlement, (ii) increased dependence on western technology, and (iii) the growing influence of foreign religions and beliefs (Decher, 1997). 
Birds constitute one of the common fauna of all habitat types, and because they are responsive to change, their diversity and abundance can reflect ecological trends in other biodiversity (Furness \& Greenwood, 1993). Because of their highly-specific habitat requirements, birds become increasingly intolerant of even slight ecosystem disturbance (Schwartz \& Schwartz, 1951). An assessment of the abundance and diversity of bird species in sacred groves can, therefore, serve as a good indication of the health of the environment in and around the grove (Bowden, 1990). Jarvinen \& Vaisenen (1978) used line-transect data on bird abundance to monitor the effect of habitat change in Nordic countries, and reported that a change in forest structure caused changes in bird populations in Northern Finish forest.

The Abiriw Sacred Grove in the Akwapim District of the Eastern Region of Ghana was partly destroyed by bushfires in 1983, resulting in loss of biodiversity. Also, because of laxity in enforcing some of the customary laws that deterred human exploitation of the forest resources, there has been increasing over-exploitation of these resources (e.g. logging, fuelwood harvesting, etc.) in the grove. The objectives of this study were, therefore, to (i) assess the abundance and diversity of the bird species inhabiting the Abiriw Sacred Grove as an indication of its current ecological status, and (ii) generate a species list of birds in the grove for conservation action and future research follow-ups.

Study area

\section{Materials and methods}

The Abiriw Sacred Grove $\left(05^{\circ} 48^{\prime} \mathrm{N} ; 00^{\circ} 06^{\prime} \mathrm{W}\right)$ is located along the Aburi-Akosombo road, about $110 \mathrm{~km}$ north of Accra in the Akuapim North District of the Eastern Region. It has an area of about $400 \mathrm{~m}^{2}$ and lies at the southern boundary of the town of Abiriw. The area falls within the moist semi-deciduous forest zone, characterized by two annual rainfall peaks. The major rainy season occurs from April to June, while the minor one occurs from September to October. Annual rainfall amounts range from $1,300 \mathrm{~mm}$ to $1,800 \mathrm{~mm}$.

The sacred grove is characterized by tree species such as Celtis adolfi-frederili, C. zenkeri, Triplochiton scleroxylon, Cylico-discus gabunensis, and Piptadenia africana. Common understory species such as Corynanthe pachyceras, Hymeno istegia afzelii, Lacaniodiscus capanicides, Momdoa myristica and Myrianthus spp. are also present. The dominant shrubs are Mussaenda erythrophylla. The brownish soil contains a lot of humus and worm cast.

The site was divided into three blocks based on the vegetation type: (i) pristine forest, a generally non-degraded core forest, (ii) forest-cultivated land boundary, outer forest sharing boundaries with cultivated land, and (iii) secondary forest, characterized by regenerated forest located between the first two blocks. Two-hour transect walks were undertaken to inventory the three blocks, with $300 \mathrm{~m}$ walk in the forest-cultivated land boundary, $210 \mathrm{~m}$ in the secondary forest, and $190 \mathrm{~m}$ in the pristine forest, using existing footpaths as transects. All birds encountered (both visually and vocally) were systematically recorded with counts repeated six times between 26th February and 14th April 2005. There were no width limit on the transects because, while the estimate of distance to visually observed birds were easier, estimate of distance to calls was more difficult, and such estimates would be highly unreliable.

\section{Analysis of data}

Bird community diversity for each block was calculated using Shannon Wiener $(\mathrm{H})$ and Simpson indices (D) while the similarities in the three blocks were calculated using Jaccard Index (Cj) given that:

$\mathrm{H}=\sum(\mathrm{ni} / \mathrm{N} *$ In ni/N) ..................

$\left.\mathrm{D}=\sum[\mathrm{ni} *(\mathrm{ni}-1) / \mathrm{N} * \mathrm{~N}-1)\right] \ldots \ldots \ldots \ldots \ldots . .2$ 
$\mathrm{Cj}=\mathrm{j} /(\mathrm{a}+\mathrm{b}-\mathrm{j}) \ldots \ldots \ldots \ldots \ldots \ldots \ldots \ldots \ldots . \ldots \ldots$

where $\mathrm{ni}=$ number of individuals in the $\mathrm{i}^{\text {th }}$ species, $\mathrm{N}=$ total number of individuals recorded, $\mathrm{j}=$ number of species found in both sites, ' $a$ ' = number of species in site ' $A$ ' and ' $b$ ' = number of species in site 'B'. The relative abundance (RA) of each species in the different blocks was also calculated.

\section{Results and discussion}

Relative abundance and species diversity There were 411 individual birds recorded, comprising 66 species belonging to 22 families. The dominant family was Muscicapidae, comprising $24.2 \%$ of the total species, followed in order by Capitonidae (12.1\%), Cuculidae (9\%), Estrildidae (7.6\%), and Pycnonotidae (7.6\%). Forty different species were recorded in the secondary forest, while 41 and 36 species were recorded in the forest cultivated land boundary and the Pristine forests, respectively (Table 1). Out of the 40 species encountered in the secondary forest, 27 individuals $(67.5 \%)$, representing eight species, were identified as pure inhabitants of forest vegetation while $20 \%$ were savanna generals. Species from other vegetation zones such as coastal thickets made up $12.5 \%$.

TABLE 1

Checklist of the birds of the Abiriw Sacred Grove, Abiriw-Akwapim

Species

Common name

$\begin{array}{ll}\begin{array}{l}\text { Occurrence in } \\ \text { blocks }\end{array} & \begin{array}{c}\text { Original } \\ \text { habitat }\end{array} \\ \text { PF SF FCLB } & \end{array}$

Muscicapidae

1. Apalis shapii

2. Camaroptera brachyura

3. C. chloronata

4. Cisticola cantans

5. C. lateralis

6. Eremomela pusilla

7. Fraseria ocreata

8. Hylia prasina

9. Macrosphenus concolor

10. M. flavicans

11. Muscicapa epulata

12. Prinia erythroptera

13. Stiprornis erythrothorax

14. Sylvietta brachyura

15. Tauraco persa

16. Terpsiphone rufiventer

Total species $=16(24.2 \%)$

Capitonidae

17. Gymnobucco calvus

18. Lybius hirsutus

19. L. vieilloti

20. Pogoniulus atro-flavus

21. P. bilineatus

22. P. scolopaceus

23. P. subsulphureus

Sharpe's apalis

Grey-backed camaroptera

Green-backed camaroptera

Singing cisticola

Whistling cisticola

Green-backed eremomela

Fraser's flycatcher

Green hylia

Olive longbill

Kemp's longbill

Little grey flycatcher

Red-winged warbler

Forest robin

Nuthatch warbler

Green-crested touraco

Red-bellied paradise flycatcher

Naked-faced barbet Hairy-breasted barbet

Vieillot's barbet

Red-rumped tinker-bird

Lemon-rumped tinker-bird

Speckled tinker-bird

Yellow-throated tinker-bird

24. Trachyphonus purpuratus Yellow-billed barbet

Total species $=8(12.1 \%)$

Cuculidae 
25. Centropus leucogaster

26. C. senegalensis

27. Ceuthmochares aereus

28. Chrysococcyx caprius

29. C. cupreus

30. C. klaas

Total species $=6(9.0 \%)$

Estrildidae

31. Estrilda melpoda

32. Lonchura bicola

33. L. cucullata

34. Nigrita canicapilla

35. N. luteifrons

Total species $=5(7.6 \%)$

Pycnonotidae

36. Andropadus curvirostris

37. A. latirostris

38. A. virens

39. Pycnonotus barbatus

40. Thescelocichla leucopleurus

Total species $=5(7.6 \%)$

Columbidae

41. Streptopelia semitorquata

42. Treron australis

43. Turtur afer

44. Turtur tympanistra

Ploceidae

45. Ploceus cucullatus

46. P. nigricollis

47. Quelea quelea

Accipitridae

48. Accipiter toussenelii

49. Nephron monachus

Bucerotidae

50. Tockus semifasciatus

51. Tropicranus albocristatus

Nectariniidae

52. Nectarinia chloropygia

53. N. olivacea

Phasianidae

54. Francolinus ahantensis

55. Ptilopachus petrosus

Alcedinidae

56. Halcyon senegalensis

Apodidae

57. Cypsiurus parvus

Coraciidae

58. Eurystoma glaucurus
Black-throated coucal

Senegal coucal

Yellowbill

Didric cockoo

Emerald cuckoo

Klass cuckoo

Orange-cheeked waxbill

Black-and-white mannikin

Bronze mannikin

Grey-crowned negro-finch

Pale-breasted negro-finch

$\begin{array}{lllll}“ & \cdots & & \mathrm{S} & \mathrm{C} \\ \cdots & \cdots & \cdots & \mathrm{S} & \mathrm{C} \\ & \cdots & \cdots & \mathrm{F} & \mathrm{C} \\ & \cdots & & \mathrm{S} & \mathrm{SC} \\ & & & \mathrm{S} & \mathrm{C} \\ & \cdots & & \mathrm{F} & \mathrm{NUR}\end{array}$

Cameroon sombre greenbul Yellow-whiskered greenbul

Little greenbul

Common garden bulbul

Swamp palm bulbul

Red-eyed dove

Green pigeon

Red-billed wood-dove

Tambourine dove

Village weaver

Spectacled weaver

Red-billed quelea

West African goshawk

Hooded vulture

Allied/Pied hornbill

White-crested hornbill

Olive-bellied sunbird

Olive sunbird

Ahanta francolin

Stone partridge

Senegal kingfisher

Palm swift

Broad-billed roller

$\begin{array}{lcl}“ & \mathrm{~S} & \mathrm{~A} \\ “ & \mathrm{FC} & \mathrm{C} \\ “ & \mathrm{~S} & \mathrm{C} \\ “ & \mathrm{~F} & \mathrm{C} \\ \cdots & \mathrm{F} & \mathrm{NU}\end{array}$

FSB NUR

$\mathrm{F} \quad \mathrm{C} / \mathrm{W}$

F A/W

$\mathrm{S} \quad \mathrm{A}$

F C/W

$\begin{array}{ccccc}“ & \cdots & \cdots & \mathrm{S} / \mathrm{FC} & \mathrm{A} \\ \cdots & \cdots & & \mathrm{F} & \mathrm{C} \\ \cdots & & \cdots & \mathrm{F} & \mathrm{C} \\ \cdots & \cdots & & \mathrm{F} & \mathrm{C}\end{array}$

“ $\quad$ F/CT NUR

" S -

(n)

F C

FS A

$\begin{array}{lllll}\text { “ } & \cdots & \text { “ } & \mathrm{F} & \mathrm{VC} \\ \cdots & \cdots & \cdots & F & N U R\end{array}$

$\begin{array}{lllll}\text { “ } & \cdots & \text { “ } & \mathrm{F} & \mathrm{VC} \\ \cdots & \cdots & \cdots & F & N U R\end{array}$

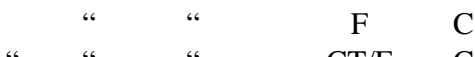

$\mathrm{CT} / \mathrm{F} \quad \mathrm{C}$

F C

S NUR

F $\quad$ C

OPP C

S C
“ $\mathrm{CFZ} \quad \mathrm{A}$ 
Corvidae

59. Corvus

Fringillidae

60. Serinus mozxambicus

Pied crow

Yellow canary

Laniidae

61. Lanius collaris

Malaconotidae

62. Tchagra senegala

Piciformes

63. Mesopicos pyrrhogaster

Platysteiridae

64. Platysteria castanea

Sturnidae

65. Lamprotornis splendidus

Zosteropidae

66. Zosteropus senegalensis

Total
Fiscal shrike

Black-crowned tchagra

Fire-bellied woodpecker

Chestnut wattle-eye

Splendid glossy starling

Yellow white-eye

\begin{tabular}{|c|c|c|c|}
\hline \multicolumn{2}{|c|}{ “ } & - & $\mathrm{C}$ \\
\hline & “ & $S$ & $\mathrm{C}$ \\
\hline & $“$ & CT & NUR \\
\hline “ & “ & $S$ & $\mathrm{C}$ \\
\hline & $“$ & $\mathrm{~F}$ & NUR \\
\hline “" & & F & $\mathrm{C}$ \\
\hline “ ' & “ & F & NUR \\
\hline & $“$ & $\mathrm{~S}$ & $\mathrm{C}$ \\
\hline 36 & 40 & 40 & \\
\hline
\end{tabular}

Legend

Plots: $\quad \mathrm{PF}=$ Pristine forest, $\mathrm{SF}=$ Secondary forest, $\mathrm{FCLB}=$ Forest-cultivated land boundary

Habitat: $\mathrm{F}=$ Forest, $\mathrm{S}=$ Savanna, $\mathrm{FSB}=$ Forest-savanna boundary, $\mathrm{FC}=$ Forest clearings, $\mathrm{CT}=\mathrm{Coastal}$ thicket, $\mathrm{CFZ}$ $=$ Coastal forest zone, $\mathrm{OPP}=$ Oil palm plantations .

Status : NUR $=$ Not uncommon resident, $\mathrm{VC}=$ Very common, $\mathrm{W}=$ Widespread $, \mathrm{C}=\mathrm{Common}, \mathrm{U}=\mathrm{Uncommon}, \mathrm{SC}$ $=$ Seasonally common, $\mathrm{A}=$ Abundant, $\mathrm{NU}=$ Not uncommon.

In the forest-cultivated land boundary, $46.3 \%$ of the species encountered were identified as forest specialists (Grimes, 1987), whilst 29.3\% were savanna generals. Olaniyan (1968) identified savanna bird species in West Africa and described them as mostly seed-eaters contrary to forest bird species which are fruit-eaters. Since most savanna birds are seed eaters, their presence in a forest area suggests trans-formation of forest vegetation into savanna. Species from other vegetation zones scored $24.4 \%$ (Table 1). About $30 \%$ of bird species in forest vegetation being savanna specialists suggests a high level of degradation. Also, the high number of bird species that are inhabitants of other vegetation zones in this area indicates that the forest is an island in 'troubled' vegetation, which served as a refuge habitat for bird species in surrounding vegetation. In the pristine forest, $66.8 \%$ of the inhabiting species were forest inhabitants, while $25 \%$ were savanna species (Table 1). However, the number of savanna species was very low in terms of individual numbers of birds in this block.

The most abundant species in the grove was the little greenbul (Andropadus virens) (RA = 8.0), followed by the bronze mannikin (Lonchura cucullata) $(\mathrm{RA}=7.8)$, common garden bulbul (Pycnonotus barbatus) $(\mathrm{RA}=7.5)$, allied hornbill (Tockus semifasciatus) $(\mathrm{RA}=6.1)$, palm swift (Cypsiurus parvus), $(\mathrm{RA}=5.4)$, and black-and-white mannikin (Lonchura bicola) $(\mathrm{RA}=4.6)$ (Table 2). These six species together make up 39.4\% of all the species recorded. The little greenbul and allied hornbill are typical forest birds, while bronze mannikin and common garden bulbul are typical savanna species. The palm swift and black-and-white mannikin occur in or near oil palm plantations and forest clearings, respectively.

TABLE 2

Numbers of birds and their relative abundance in Abiriw Sacred Grove, Abiriw-Akwapim

\begin{tabular}{|c|c|c|c|c|c|c|c|c|c|}
\hline \multirow[t]{3}{*}{ Species } & \multirow[t]{3}{*}{ Family } & \multicolumn{5}{|c|}{ Habitats } & \multirow{3}{*}{\multicolumn{2}{|c|}{$\begin{array}{l}\quad \text { Total } \\
\text { individualsRA } \\
R A\end{array}$}} & \multirow[t]{3}{*}{ Overall } \\
\hline & & \multicolumn{2}{|c|}{$P F$} & \multicolumn{3}{|c|}{$S F F C L B$} & & & \\
\hline & & Ind & $R A$ & Ind & $R A$ & Ind & & & \\
\hline 1. Andropadus virens & Pycnonotidae & 8 & 7.2 & 12 & 13.5 & 13 & 6.2 & 33 & 8.0 \\
\hline 2. Lonchura cucullata & Estrildidae & - & - & - & - & 32 & 15.2 & 32 & 7.8 \\
\hline
\end{tabular}




\begin{tabular}{|c|c|c|c|c|c|c|c|c|c|c|}
\hline 3. & Pycnonotus barbatus & Pycnonotidae & 3 & 2.7 & 2 & 2.3 & 26 & 12.3 & 31 & 7.5 \\
\hline 4. & Tockus semifasciatus & Bucerotidae & 9 & 8.1 & 6 & 6.7 & 10 & 4.5 & 25 & 6.1 \\
\hline 5. & Cypsiurus parvus & Apodidae & - & - & - & - & 22 & 10.4 & 22 & 5.4 \\
\hline 6. & Lonchura bicola & Estrildidae & - & - & - & - & 19 & 9.0 & 19 & 4.6 \\
\hline 7. & Hylia prasina & Muscicapidae & 9 & 8.1 & 6 & 6.7 & 1 & 0.5 & 16 & 3.9 \\
\hline 8. & Tauraco persa & Muscicapidae & 8 & 7.2 & 4 & 4.5 & - & - & 12 & 2.9 \\
\hline 9. & Nigrita canicapilla & Estrildidae & 4 & 3.6 & 2 & 2.3 & 4 & 1.9 & 10 & 2.4 \\
\hline 10. & Pogoniulus subsulphureus & Capitonidae & 3 & 2.7 & 4 & 4.5 & 2 & 1.0 & 9 & .2 \\
\hline 11. & Zosteropus senegalensis & Zosteropidae & - & - & - & - & 9 & 4.3 & 9 & 2.2 \\
\hline 12. & Nectarinia olivacea & Nectariniidae & 2 & 1.8 & 2 & 2.3 & 4 & 1.9 & 8 & 1.9 \\
\hline 13. & Camaroptera brachyura & Muscicapidae & 1 & 0.9 & 2 & 2.3 & 5 & 2.4 & 8 & 1.9 \\
\hline 14. & Francolinus ahantensis & Phasianidae & 6 & 5.4 & 2 & 2.3 & - & - & 8 & 1.9 \\
\hline 15. & Centropus senegalensis & Cuculidae & 1 & 0.9 & 1 & 1.1 & 5 & 2.4 & 7 & 1.7 \\
\hline 16. & Sylvietta brachyura & Muscicapidae & 4 & 3.6 & 1 & 1.1 & 2 & 1.0 & 7 & 1.7 \\
\hline 17. & Trachyphonus purpuratus & Capitonidae & 4 & 3.6 & 3 & 3.4 & - & - & 7 & 1.7 \\
\hline 18. & Pogoniulus scolopaceus & Capitonidae & 2 & 1.8 & 1 & 1.1 & 3 & 1.4 & 6 & 1.5 \\
\hline 19. & P. bilineatus & Capitonidae & 2 & 1.8 & 1 & 1.1 & 3 & 1.4 & 6 & 1.5 \\
\hline 20. & Centropus leucogaster & Cuculidae & 2 & 1.8 & 4 & 4.5 & - & - & 6 & 1.5 \\
\hline 21. & Nigrita luteifrons & Estrildidae & 1 & 0.9 & - & - & 5 & 2.4 & 6 & 1.5 \\
\hline 22. & Thescelocichla & $\begin{array}{l}\text { Pycnonotidae } \\
\text { leucopleurus }\end{array}$ & 6 & 5.4 & - & - & - & - & 6 & 1.5 \\
\hline 23. & Corvus albus & Corvidae & - & - & - & - & 6 & 2.8 & 6 & 1.5 \\
\hline 24. & Ceuthmochares aereus & Cuculidae & 2 & 1.8 & 2 & 2.3 & 1 & 0.5 & 5 & 1.2 \\
\hline 25. & Treron australis & Columbidae & 4 & 3.6 & 1 & 1.1 & - & - & 5 & 1.2 \\
\hline 26. & Streptopelia semitorquata & Columbidae & 2 & 1.8 & 2 & 2.3 & 1 & 0.5 & 5 & 1.2 \\
\hline 27. & Nephron monachus & Accipitricidae & - & - & 3 & 3.4 & 2 & 1.0 & 5 & 1.2 \\
\hline 28. & Tropicranus albocristatus & Bucerotidae & 3 & 2.7 & 1 & 1.1 & 1 & 0.5 & 5 & 1.2 \\
\hline 29. & Nectarinia chloropygia & Nectariniidae & - & - & 2 & 2.3 & 3 & 1.4 & 5 & 1.2 \\
\hline 30. & Lamprotornis splendidus & Sturnidae & - & - & 3 & 3.4 & 2 & 1.0 & 5 & 1.2 \\
\hline 31. & Ptilopachus petrosus & Phasianidae & 5 & 4.5 & - & - & - & - & 5 & 1.2 \\
\hline 32. & Andropadus latiroster & Pycnonotidae & 2 & 1.8 & 2 & 2.3 & - & - & 4 & 1.0 \\
\hline 33. & Lybius hirsutus & Capitonidae & 2 & 1.8 & 1 & 1.1 & 1 & 0.5 & 4 & 1.0 \\
\hline 34. & Turtur tympanistra & Columbidae & 3 & 2.7 & 1 & 1.1 & - & - & 4 & 1.0 \\
\hline 35. & Camaroptera chloronata & Muscicapidae & 1 & 0.9 & 2 & 2.3 & - & - & 3 & 0.7 \\
\hline 36. & Pogoniulus atro-flavus & Capitonidae & - & - & 1 & 1.1 & 2 & 1.0 & 3 & 0.7 \\
\hline 37. & Gymnobucco calvus & Capitonidae & - & - & 1 & 1.1 & 2 & 1.0 & 3 & 0.7 \\
\hline 38. & Estrilda melpoda & Estrildidae & - & - & - & - & 3 & 1.4 & 3 & 0.7 \\
\hline 39. & Ploceus cucullatus & Ploceidae & - & - & - & - & 3 & 1.4 & 3 & 0.7 \\
\hline 40. & Quelea quelea & Ploceidae & - & - & - & - & 3 & 1.4 & 3 & 0.7 \\
\hline 41. & Tchagra senegala & Malaconotidae & - & - & 1 & 1.1 & 2 & 1.0 & 3 & 0.7 \\
\hline 42. & Cisticola cantans & Muscicapidae & - & - & - & - & 2 & 1.0 & 2 & 0.5 \\
\hline 43. & C. lateralis & Muscicapidae & - & - & - & - & 2 & 1.0 & 2 & 0.5 \\
\hline 44. & Eremomela pusilla & Muscicapidae & 2 & 1.8 & - & - & - & - & 2 & 0.5 \\
\hline 45. & Stiprornis erythrothorax & Muscicapidae & 2 & 1.8 & - & - & - & - & 2 & 0.5 \\
\hline 46. & Fraseria ocreata & Muscicapidae & - & - & 2 & 2.3 & - & - & 2 & 0.5 \\
\hline 47. & Macrosphenus flavicans & Muscicapidae & 1 & 0.9 & 1 & 1.1 & - & - & 2 & 0.5 \\
\hline 48. & Muscicapa epulata & Muscicapidae & - & - & - & - & 2 & 1.0 & 2 & 0.5 \\
\hline 49. & Prinia erythroptera & Muscicapidae & - & - & - & - & 2 & 1.0 & 2 & 0.5 \\
\hline 50. & Terpsiphone rufiventer & Muscicapidae & - & - & 2 & 2.3 & - & - & 2 & 0.5 \\
\hline 51. & Turtur afer & Columbidae & 1 & 0.9 & - & - & 1 & 0.5 & 2 & 0.5 \\
\hline 52. & Ploceus nigricollis & Ploceidae & - & - & - & - & 2 & 1.0 & 2 & 0.5 \\
\hline 53. & Halcyon senegalensis & Alcedinidae & - & - & 1 & 1.1 & 1 & 0.5 & 2 & 0.5 \\
\hline 54. & Eurystoma glaucurus & Coraciidae & - & - & 2 & 2.3 & - & - & 2 & 0.5 \\
\hline & Lanius collaris & Laniidae & - & - & - & - & 2 & 1.0 & 2 & 0.5 \\
\hline 56. & Apalis shapii & Muscicapidae & 1 & 0.9 & - & - & - & - & 1 & 0.2 \\
\hline & Macrosphenus concolor & Muscicapidae & - & - & 1 & 1.1 & - & - & 1 & 0.2 \\
\hline & Lybius vieilloti & Capitonidae & 1 & 0.9 & - & - & - & - & 1 & \\
\hline 59. & Chrysococcyx caprius & Cuculidae & - & - & 1 & 1.1 & - & - & 1 & 0.2 \\
\hline
\end{tabular}




$\begin{array}{llllllllll}\text { 60. C. cupreus } & \text { Cuculidae } & - & - & 1 & 1.1 & - & - & 1 & 0.2 \\ \text { 61. C. klaas } & \text { Cuculidae } & - & - & 1 & 1.1 & - & - & 1 & 0.2 \\ \text { 62. Andropadus curvirostria } & \text { Pycnonotidae } & - & - & 1 & 1.1 & - & - & 1 & 0.2 \\ \text { 63. Accipiter toussenelii } & \text { Accipitridae } & 1 & 0.9 & - & - & - & - & 1 & 0.2 \\ \text { 64. Serinus mozxambicus } & \text { Fringillidae } & - & - & - & - & 1 & 0.5 & 1 & 0.2 \\ \text { 65. Mesopicos pyrrhogaster } & \text { Picidae } & - & - & - & - & 1 & 0.5 & 1 & 0.2 \\ \text { 66. Platysteria castanea } & \text { Platysteiridae } & - & - & 1 & 1.1 & - & - & 1 & 0.2 \\ \text { Total } & & 108 & & & & & & & \end{array}$

Legend

$\mathrm{PF}=$ Pristine forest, $\mathrm{SF}=$ Secondary forest, FCLB = Forest-cultivated land boundary, Ind = Number of individuals, RA $=$ Relative abundance

\section{Species richness and representation}

Eighty nine individual birds were encountered in the secondary forest, while 111 and 211 individuals were recorded in the pristine forest and forest-cultivated land boundary, respectively. Shannon-Wiener indices $(\mathrm{H})$ of $0.012,0.011$ and 0.012 were obtained for the secondary forest, forest-cultivated land boundary and the pristine forest, respectively. The reverse Simpson index (i.e. 1-D) yielded values of $0.99,0.93$ and 0.96 for the secondary forest, forest-cultivated land boundary and the pristine forest, respectively, suggesting that the secondary forest had the highest bird species diversity. This is further supported by the values for the Shannon -Wiener index (Table 3). The Jaccard Index of Similarity (Cj) between the secondary and pristine forest yielded a value of 0.46 , while the similarity between the secondary forest and the forest- cultivated land boundary was 0.36 . The pristine forest and forest-cultivated land boundary had a similarity of 0.29 . Since a Jaccard index of ' 1 ' implies complete similarity and ' 0 ' denotes complete dissimilarity, the values revealed little or no similarity between the blocks in terms of bird usage. Since the habitat choice of birds is highly specific (Schwartz \& Schwartz, 1951) and driven by plant communities, variations in bird communities for the various blocks suggests different habitat types which could be reflecting different levels of habitat degradation.

TABLE 3

Major bird families recorded in different habitats at Abiriw Sacred Grove

\begin{tabular}{|c|c|c|c|c|c|c|c|c|c|c|c|c|}
\hline \multirow[t]{2}{*}{ Family } & \multicolumn{4}{|c|}{$P F$} & \multicolumn{4}{|c|}{$S F$} & \multicolumn{4}{|c|}{$F C L B$} \\
\hline & $S p$ & $\%$ & Ind & $\%$ & $S p$ & $\%$ & Ind & $\%$ & $S p$ & $\%$ & Ind & $\%$ \\
\hline Muscicapidae & 9 & 25 & 29 & 25.0 & 7 & 17.5 & 15 & 16.9 & 7 & 17.1 & 16 & 7.6 \\
\hline Capitonidae & 7 & 19.4 & 16 & 19.4 & 7 & 17.5 & 12 & 13.5 & 5 & 12.2 & 11 & 5.2 \\
\hline Cuculidae & 4 & 11.1 & 6 & 11.1 & 5 & 12.5 & 9 & 10.1 & 2 & 4.9 & 6 & 2.8 \\
\hline Estrildidae & & & & & & & & & 5 & 12.2 & 63 & 29.9 \\
\hline Pycnonotidae & 4 & 11.1 & 19 & 11.1 & 4 & 10.0 & 17 & 19.1 & 2 & 4.9 & 39 & 18.5 \\
\hline Columbidae & 4 & 11.1 & 10 & 11.1 & 3 & 7.5 & 4 & 4.5 & 2 & 4.9 & 2 & 1.0 \\
\hline Ploceidae & & & & & & & & & 2 & 4.9 & 5 & 2.4 \\
\hline Bucerotidae & 2 & 5.6 & 12 & 5.6 & 2 & 5.0 & 7 & 7.9 & 2 & 4.9 & 11 & 5.2 \\
\hline Nectariniidae & & & & & 2 & 5.0 & 4 & 4.5 & 2 & 4.9 & 7 & 3.3 \\
\hline TOTAL & 30 & & 92 & & 30 & & 68 & & 29 & & 160 & \\
\hline H (Shannon-Wiener) & 0.012 & 0.012 & 0.011 & & & & & & & & & \\
\hline 1-D (Reverse Simpson & 0.96 & 0.99 & 0.93 & & & & & & & & & \\
\hline
\end{tabular}

\section{Conclusion and recommendations}


The study established that the Abiriw Sacred Grove harbours a sizable number of bird species from savanna and other vegetation zones such as coastal thickets, even the primarily vegetation is forest. The traditional norms and taboos which previously prevented people from exploiting forest resources and products from the grove appeared to have been relaxed, resulting in general forest degradation. This adversely affected forest bird species and also opened up the forest for habitation by bird species from other habitats. Similarity indices indicated a trend of fragmentation in the grove, with the various fragments at different levels of degradation.

It is recommended that a buffer zone of about $20 \mathrm{~m}$ should be created along the boundaries of the grove to prevent encroachment by estate developers who build too close to the grove. Furthermore, existing regulations should be vigorously enforced by the traditional authorities in the area, and new ones introduced, with severe sanctions applied to offenders to serve as a deterrent. Traditional award ceremonies should be instituted as one of the highlights of the annual yam festival to serve as incentives to individuals and organizations which contribute to biodiversity initiatives involving the grove.

\section{Acknowledgement}

The authors are very grateful to the people of Abiriw-Akwapim, especially Nana Yaw Ohenaku and Nana Ansah Sasraku, Abusuapanyin and Gyasehene, respectively. Special thanks also go to Mr Koranteng, a hunter, for placing his expertise at the authors' disposal during fieldwork. $\mathrm{Mr}$ Michael Asoah and Nana Gyamera Afful also deserve special mention.

\section{References}

Adarkwa-Dadzie A. (1997). The Contribution of Ghanaian Beliefs to Biodiversity Conservation. Paper Presentation, UNESCO-MAB Seminar on Biosphere Reserves for Biodiversity Conservation and Sustainable Development in Anglophone Africa (BRAAF), Cape Coast. Ghana.

Biodiversity Support Program (BSP) (1993). Indigenous Knowledge and Biodiversity. African Biodiversity Foundation for the Future, Washington D.C. 149 pp.

Bowden C. G. R. (1990). Selection of foraging habitat by woodlarks nesting in pin plantation. Appl. Ecol. 27: 410419.

Campbell M. O. (2004). Traditional forest protection and woodlots in the coastal savannah of Ghana. Envir. Conserv. 31(3): 225-232.

Decher J. (1997). Conservation, small mammals, and the future of sacred groves in West Africa. Biodiv. Conserv. 6: 1007-1026.

Dorm-Adjobu C., Ampadu-Adjei O. and Veit P. G. (1991). Religious beliefs and environmental protection: the Malshegu Sacred Grove in northern Ghana. World Resources Institute, Washington DC, African Centre for Technology Studies (ACTS) Press, Nairobi, Kenya.

Furness R. W. and Greenwood J. J. D. (1993). Birds as a Monitor of Environmental Change. Chapman and Hall, London.

Gordon C. (1992). Sacred groves and conservation in Ghana. Newsletter of the IUCN SSC African Reptile and Amphibian Specialist Group 1: 3-4.

Grimes L.G. (1987). The Birds of Ghana: An Annotated Checklist. Checklist 9. British Ornithologists' Union (BOU), Tring.

Jarvinen O. and Vaisenen R. A. (1978). Recent changes in forest bird population in Northern Finland. Anim. Zool. 15: $279-289$.

Kingdon J. (1989). Island Africa: The Ecology of Africa's Rare Animals and Plants. Princeton University Press, Princeton.

Ntiamoa-Baidu Y. (1995). Indigenous versus Introduced Biodiversity Conservation Strategies: The Case of Protected Area Systems in Ghana. Biodiversity Support Programme (Issues in African Biodiversity No. 1). Washington DC.

Olaniyan C. I. O. (1968) West African Animal Ecology. Heineman Educational Books, London.

Schwartz C. W. and Schwartz E.R. (1951). An ecological reconnaissance of the pheasants of Hawaii. Auk. 68: 281314. 